\title{
Enhancement of conditioned fear during extinction
}

\author{
NICHOLAS R. WHITE and PAUL T. P. WONG \\ Trent University, Peterborough, Ontario K9J 7B8, Canada
}

\begin{abstract}
Eight rats were given five sessions each of noncontingent shock at 2-, 3-, and 4-min intervals, followed by five sessions of extinction. The rats were immobile longer during the first two sessions of extinction than they were during the final two sessions of fear conditioning. The incubation effect was attributed to the prospective function as distinct from the retrospective function of the CS.
\end{abstract}

Eysenck (1979) has suggested that, under some circumstances, animals undergoing extinction may show higher levels of the previously reinforced response than they did during conditioning (i.e., the incubation effect). Noting that some neurotic behaviors are highly resistant to extinction, Eysenck theorized that aversive CSs may acquire drive properties of their own that maintain CRs in the absence of the UCS.

In a recent review, Mineka (1979) has argued that extinction of fear is at least partially independent of extinction of avoidance-escape responding. Several studies have suggested that subjects may exhibit fear even when avoidance responding has ceased following extinction or flooding (Coulter, Riccio, \& Page, 1969; Kamin, Brimer, \& Black, 1963; Mineka \& Gino, 1979; see also Starr \& Mineka, 1977). These findings suggest that levels of conditioned fear may be higher than might be expected on the basis of avoidance response data. In addition, Mineka and Gino (1979) have suggested that conditioned fear may peak at the time that the subject ceases to respond during extinction.

Several studies have directly shown that fear levels were enhanced if the subjects experienced a brief, unreinforced CS presentation after acquisition but before a series of regularly presented extinction or flooding trials (Linton, Riccio, Rohrbaugh, \& Page, 1970; Rohrbaugh \& Riccio, 1970; Rohrbaugh, Riccio, \& Arthur, 1972). However, only in the Rohrbaugh et al. (1972) study was the total time spent in the training apparatus equated for all groups; and in this study the enhancement effect seemed rather transitory. Furthermore, in all three studies, only one brief acquisition training session was given, followed quickly by a brief unreinforced CS presentation and the other extinction or flooding trials. Recently, Boyd (1981) reported that incubation was partially related to shock intensity and that avoidance

Nicholas R. White is now at the University of Toronto. The study was supported by a research grant from the Natural Science and Engineering Research Council of Canada to the second author. behavior was increased only for high-shock groups despite prolonged exposure to nonreinforced CS.

The main purpose of the present study was to determine whether conditioned fear would be enhanced during extinction after a prolonged period of noncontingent shock presentation. Conditioned fear was measured by amount of time spent in freezing or immobility. In addition, we attempted to determine if food-deprived rats would show a higher level of fear than nondeprived rats.

\section{METHOD}

\section{Subjects}

Eight male Walker-Walker hooded rats were used in this study. All subjects were experimentally naive and had been born and reared in the Trent University vivarium. They were approximately 100 days old at the time of testing. Four of the rats were maintained at $80 \%$ of free-feeding weight, and the other four were fed ad lib.

\begin{abstract}
Apparatus
The animals were tested in a $20 \times 20 \times 22 \mathrm{~cm}$ Skinner box constructed from sheet metal and Plexiglas. The floor consisted of 12 1.4-mm steel rods connected to a BRS-Forringer shock generator and scrambler that produced a $1.0-\mathrm{mA}$ shock for 1 sec. Shock presentations were timed automatically through a tape reader.
\end{abstract}

\section{Procedure}

All rats were given three sessions of baseline training, during which no shocks were administered, followed by five sessions of fixed-time (FT) noncontingent shock presentation at each of 2-, 3-, and 4-min intervals. Finally, five sessions of extinction were given. All sessions were of $20 \mathrm{~min}$ duration and occurred on consecutive days. The experimenter observed each subject and activated solid state recording equipment when freezing occurred. Freezing was defined by complete immobility with at least three feet on the floor.

\section{RESULTS AND DISCUSSION}

Figure 1 depicts the mean time of freezing during FT 2-, 3-, and 4-min schedules, as well as during each of the five extinction sessions. The incubation effect is quite clear, in that rats showed more freezing during the 


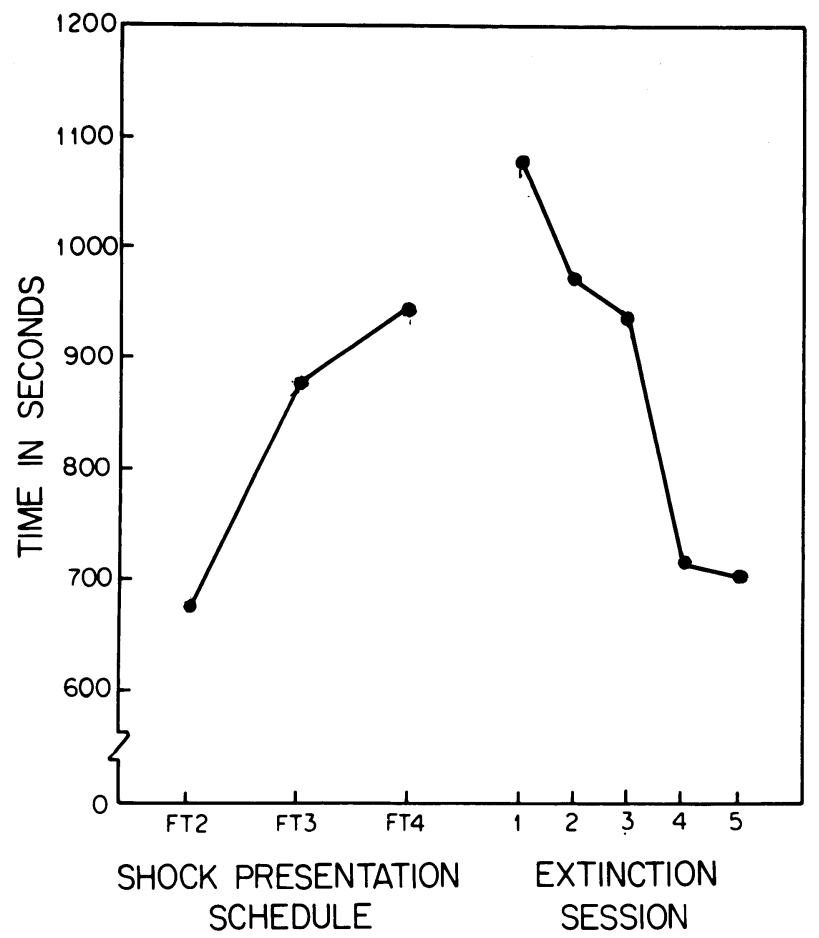

Figure 1. The mean freezing time in each schedule, FT 2, 3 , and $4 \mathrm{~min}$, and in each session of extinction.

first two sessions of extinction than during fear conditioning. Analysis of variance on the log-transformed data of the last two sessions of FT $4 \mathrm{~min}$ and the first two sessions of extinction supports the observation of more freezing during initial extinction than during terminal fear conditioning $[F(1,54)=7.35, p<.01]$. It should be noted that all eight subjects showed more freezing in the first extinction session than during FT $4 \mathrm{~min}$, and three of the eight rats continued to exhibit more freezing reponse throughout the entire course of extinction than during terminal acquisition. Thus, we have demonstrated a robust incubation effect, and the effect was quite durable in some subjects.

The present results are consistent with those of Rohrbaugh and Riccio (1970) and Rohrbaugh et al. (1972) even though a very different paradigm was used. In the present study, the CS consisted of temporal and environmental cues associated with shock. Thus Eysenck's (1979) conclusion that a strong driveproducing UCS and a short CS presentation are essential for the incubation effect needs to be modified.

The present results, especially the maintenance of a high level of conditioned fear throughout extinction in three rats, seem to support Eysenck's (1979) conditioning model of neuroses. However, when the three rats were given five additional extinction sessions, their freezing time eventually decreased to below $800 \mathrm{sec}$. After reviewing all the evidence on incubation, Wong (1979) concluded that "the empirical basis of Eysenck's model is not particularly impressive. At present, there is no unequivocal evidence that conditioned fear increases to some asymptotic level and stays there in spite of prolonged extinction" (p. 185).

From our point of view, a CS has at least two functions: retrospective and prospective. In its retrospective role, the CS functions as a retrieval cue, reminding the subject what has happened. In its prospective role, the CS functions as a signal telling the subject what will happen. Both functions may be operating simultaneously; however, we hypothesize that the prospective role should be predominant during the early stage of extinction, when anticipation of reinforcement, whether positive or negative, remains strong. However, with repeated extinction trials, the retrospective role should become predominant, when the subject no longer expects reinforcement but still remembers, however vaguely, that reinforcement has occurred in the presence of the CS.

The present incubation effect could be attributed to the prospective function of the CS. Although we did not use a distinct CS, both the physical and temporal cues associated with being placed inside the Skinner box predicted shock onset, and shock offset provided a discriminative cue signaling a brief safety period. (During fear conditioning training, a decrease in freezing was consistently observed immediately following the shock.) During the early stage of extinction, rats anticipated shock and the temporal relief that comes after it. They continued to freeze when shock did not occur at the anticipated time: hence, the increase in freezing. With prolonged extinction, rats no longer anticipated shock, but the memory of prior shock should still have evoked a certain amount of conditioned fear.

Future research should attempt to identify the important variables that have different effects on retrospective fear and prospective fear. It is likely that these two kinds of conditioned fear have different properties and obey different laws.

In the present study, neither the deprivation main effect nor the Deprivation by Schedule interaction was significant during fear conditioning. Also, food deprivation had no effect on extinction trials. Thus, there is no evidence that food deprivation makes the rats more susceptible to conditioned fear.

\section{REFERENCES}

Boyd, T. L. The effects of shock intensity on fear incubation (enhancement): A preliminary investigation of Eysenck's theory. Behavioral Research and Therapy, 1981, 19, 413-418.

Coulter, X., Riccio, D. C., \& PAge, H. A. Effects of blocking an instrumental avoidance response: Facilitated extinction but persistence of fear. Journal of Comparative and Physiological Psychology, 1969, 68, 377-381.

EysenCK, H. J. The conditioning model of neurosis. Behavioral and Brain Sciences, 1979, 2, 155-199.

Kamin, L. J., Brimer, C. J., \& Black, A. H. Conditioned 
suppression as a monitor of fear of the CS in the course of avoidance training. Journal of Comparative and Physiological Psychology, 1963, 56, 497-501.

Linton, J. Riccio, D. C., Rohrbaugh, M., \& Page, H. A. The effects of blocking an instrumental avoidance response: Fear reduction or enhancement? Behavior Research and Therapy, 1970, 8, 267-272.

Mine KA, S. The role of fear in theories of avoidance learning, flooding, and extinction. Psychological Bulletin, 1979, 86, 985-1010.

MinekA, S., \& Gino, A. Dissociative effects of different types and amounts of nonreinforced CS exposure on avoidance extinction and the CER. Learning and Motivation, 1979, 10, 141-160.
Rohrbaugh, M., \& Riccio, D. C. Paradoxical enhancement of learned fear. Journal of Abnormal Psychology, 1970, 75, 210-216.

Rohrbaugh, M., Riccio, D. C., \& Arthur, A. Paradoxical enhancement of conditioned suppression. Behavior Research and Therapy, 1972, 10, 125-130.

StarR, M. D., \& Mineka, S. Determinants of fear over the course of avoidance learning. Learning and Motivation, 1977, 8, 332-350.

Wong, P. T. P. A critique of Eysenck's theory of neurosis. Behavioral and Brain Sciences, 1979, 2, 185-186.

(Received for publication September 28, 1982.) 